\title{
DAF-18/PTEN locally antagonizes insulin signalling to couple germline stem cell proliferation to oocyte needs in $C$. elegans
}

\author{
Patrick Narbonne $^{1, *}$, Paul S. Maddox ${ }^{1,2}$ and Jean-Claude Labbé ${ }^{1}$
}

\begin{abstract}
During development, stem cell populations rapidly proliferate to populate the expanding tissues and organs. During this phase, nutrient status, by systemically affecting insulin/IGF-1 signalling, largely dictates stem cell proliferation rates. In adults, however, differentiated stem cell progeny requirements are generally reduced and vary according to the spatiotemporal needs of each tissue. We demonstrate here that differential regulation of germline stem cell proliferation rates in Caenorhabditis elegans adults is accomplished through localized neutralization of insulin/IGF-1 signalling, requiring DAF-18/PTEN, but not DAF-16/FOXO. Indeed, the specific accumulation of oocytes, the terminally differentiated stem cell progeny, triggers a feedback signal that locally antagonizes insulin/ IGF-1 signalling outputs in the germ line, regardless of their systemic levels, to block germline stem cell proliferation. Thus, during adulthood, stem cells can differentially respond within tissues to otherwise equal insulin/IGF-1 signalling inputs, according to the needs for production of their immediate terminally differentiated progeny.
\end{abstract}

KEY WORDS: C. elegans, Feedback regulation, Germline stem cells, Insulin signalling, Oocyte accumulation, Proliferation

\section{INTRODUCTION}

A variety of adult stem cells exist in multicellular organisms to fulfil the specific homeostatic needs of each tissue. Some tissues, such as the gastrointestinal epithelium, have a rapid cellular turnover rate and, accordingly, their stem cells self-renew and differentiate more frequently than those of tissues that turnover more slowly, such as the bone (Tomasetti and Vogelstein, 2015). The requirements of differentiated stem cell progeny may also vary spatiotemporally within tissues, for example following a localized injury. We aimed to understand how adult stem cell activity is locally regulated when information about nutrient status, which largely influences stem cell proliferation rates across all tissues (Narbonne and Roy, 2006a; Shim et al., 2013), is regulated at a systemic level (Murphy and Hu, 2013; Murphy et al., 2007).

Stem cell activities (self-renewal and differentiation) are controlled by a variety of intrinsic and extrinsic factors. To maintain their self-renewal capacities, all stem cells require signalling from neighbouring niche cells, which act to prevent differentiation. If stem cells move outside of the niche signal range, or if niche signalling is impaired, the stem cells switch to their

\footnotetext{
${ }^{1}$ Département de Pathologie et Biologie Cellulaire, Institut de Recherche en Immunologie et en Cancérologie (IRIC), Université de Montréal, Montréal, Québec, Canada H3T 1J4. ${ }^{2}$ Department of Biology, University of North Carolina, Chapel Hill, NC 27599, USA.

*Author for correspondence (patrick.narbonne@umontreal.ca)
}

Received 10 September 2015; Accepted 29 October 2015 default fate and differentiate (Morrison and Kimble, 2006). Thus, niche signalling primarily governs stem cell fate, and as a consequence, the size of the niche and the nature and extent of niche signalling have a large influence on the size of stem cell populations. Stem cells are also affected by growth factors that impinge on their proliferation rates, largely independently of niche signalling. Nutrient intake, as well as systemic insulin/IGF-1 signalling (IIS) levels, dictate germline stem cell (GSC) proliferation rates in C. elegans and Drosophila (LaFever and Drummond-Barbosa, 2005; Narbonne and Roy, 2006a; Shim et al., 2013). In C. elegans, if nutrients are depleted from the environment and/or if IIS is downregulated, GSCs dramatically reduce their proliferation rates and can enter a quiescent state (Angelo and Van Gilst, 2009; Michaelson et al., 2010; Narbonne and Roy, 2006b). Conversely, if IIS cannot be downregulated when nutrients are depleted, such as in the absence of DAF-18/PTEN (phosphatase and tensin homologue), a phosphatidylinositol 3-phosphate phosphatase that counteracts the activity of PI3K (phosphatidylinositol 3phosphate kinase) downstream of the insulin/IGF-1 receptor, GSC quiescence is not properly induced or maintained (Fukuyama et al., 2006; Narbonne and Roy, 2006b; Ogg and Ruvkun, 1998). Thus, niche signalling governs stem cell fate (self-renewal or differentiation) and regulates pool size, whereas growth factors, largely in parallel, dictate the rate at which stem cells will proliferate.

Stem cell activities also tend to decline during ageing (López-Otín et al., 2013). In adult mice for example, muscle stem cells (also known as satellite cells) are quiescent and become harder to reactivate following injury owing to an age-dependent change in blood-borne factors (Conboy et al., 2005). These cells also intrinsically acquire changes during ageing, which lead to irreversible senescence in very old (geriatric) mice (Sousa-Victor et al., 2014). Stem cell population sizes also decline during ageing (López-Otín et al., 2013), and a recent study (Qin and Hubbard, 2015) provided evidence that the size of the GSC population in ageing $C$. elegans adults is nonautonomously controlled by IIS in the proximal somatic gonad, in a manner dependent on DAF-16, the $C$. elegans FOXO orthologue (Lee et al., 2001; Lin et al., 2001). However, the kinetics of the decline in the GSC population, as well as the mechanisms that regulate stem cell proliferation rates during ageing remain unclear.

The germ line of an adult $C$. elegans hermaphrodite consists of two gonad arms, each with 200 GSCs at its distal tip (Fig. S1A). At each tip resides a niche that consists of a single cell, the distal tip cell (DTC), which locally prevents GSC differentiation through a Notch signal (Austin and Kimble, 1987). As GSCs proliferate, some consequently move away from the DTC, and the Notch signal they receive thus presumably progressively declines, eventually reaching a threshold that allows meiotic differentiation (Fox and Schedl, 2015; Hansen and Schedl, 2013). In this article, the term 'GSCs' refers to the more or less homogeneous pool of undifferentiated germ cells (Fox and Schedl, 2015; Gerhold et al., 2015). During the differentiation process, germ cells sequentially progress through 
meiotic entry, pachytene, diplotene and diakinesis stages, thus progressively transforming into oocytes (Zetka, 2009). As such, the adult $C$. elegans germ line can be viewed as a biological assembly line, with stem cells as the raw material that is turned, in a stepwise manner, into mature oocytes. Under replete growth conditions, hermaphrodite larvae first produce $\sim 300$ sperm cells, before switching to exclusive production of oocytes, which is maintained during adulthood. The first $\sim 300$ oocytes produced are thus selffertilized, after which oocyte maturation is inhibited and unfertilized oocytes begin to accumulate in the proximal gonad. An aged spermdepleted hermaphrodite will, however, reinitiate oocyte maturation after mating with a male (Kimble and Ward, 1988). The stimulation of oocyte maturation by the presence of sperm is mediated by sperm-secreted major sperm proteins (MSPs) which, through cAMP signalling in the sheath cells of the proximal somatic gonad, induce the final maturation of the proximal oocyte (Govindan et al., 2009; Miller et al., 2001). Interestingly, adult hermaphrodites carrying a mutation that feminizes the germ line $(f \circ g-1)$, and thus prevents sperm formation, were reported to have lower rates of stem cell proliferation, and this phenotype was reversed when the animals were provided with sperm (Morgan et al., 2010). How GSC proliferation rates may be adjusted in response to sperm availability in ageing adults is not known.

Here, we used the GSCs of the nematode $C$. elegans to improve our understanding of the physiological mechanisms that underlie the observed decline in adult stem cell activity in ageing individuals, in order to uncover how differential stem cell regulation might be achieved. We found that the proliferative activity of these adult stem cells declines according to two parameters: the needs for differentiated GSC progeny and a separate mechanism that probably results from the detrimental effects of ageing. We found that self-sperm depletion in adult hermaphrodites triggers a feedback mechanism that requires DAF-18 to suppress GSC activities. We show that downstream of self-sperm depletion, an arrest in oocyte maturation and/or the resulting accumulation of oocytes, leads to the inhibition of GSC proliferation in a gonad arm-autonomous manner. We also show that canonical IIS levels influence the rates of GSC proliferation in C. elegans adults: if IIS is systemically inhibited, GSCs proliferate slower, whereas if it is activated, GSCs proliferate more rapidly. We further show that canonical IIS needs to be downregulated in the germ line in order for the GSCs to slow down their proliferation rates when food is available. Thus, we conclude that DAF-18 can locally antagonize IIS to arrest oocyte maturation and block GSC proliferation specifically in one sperm-depleted gonad arm.

\section{RESULTS GSC proliferation and differentiation activities decline with age}

To assess how GSC activities are affected during ageing, we first evaluated the level of GSC proliferation in unmated ageing adult hermaphrodites. GSC proliferation rates can be evaluated based on their mitotic index (MI), which is obtained by dividing the number of M-phase GSCs by the total number of GSCs (Crittenden et al., 2006). This can be used because variation in M-phase duration is largely due to spindle assembly checkpoint activity and not age or growth condition as is the case for interphase duration, and thus cells that divide frequently are more often in $\mathrm{M}$ phase and have a high MI (Gerhold et al., 2015; Morris et al., 1990). We used a nuclear marker (DAPI), an M-phase marker (anti-phospho-H3ser10 antibody) (Hendzel et al., 1997) and a differentiation marker (anti-HIM-3 antibody) (Zetka, 2009) to label all germ cells, M-phase GSCs and their differentiated progeny, respectively (Fig. 1A-D). In unmated hermaphrodites, the GSC MI, and thereby their proliferative activity, was maximal during larval stages, then steadily declined during the first 3 days of adulthood reaching a steady-state basal level from adult day 4 (A4). This basal level was thereafter seemingly maintained at least until A10 (Fig. 1A,C,E). A10 corresponds to $83 \%$ of $C$. elegans' life expectancy at $25^{\circ} \mathrm{C}$, and is well beyond reproductive cessation in unmated and mated animals (data not shown). GSC pool size peaked at A2, with $\sim 180 \mathrm{GSCs} /$ gonad arm, then decreased to $\sim 100$ GSCs by A6, and essentially remained stable thereafter (Fig. 1E), consistent with published data (Qin and Hubbard, 2015). The number of M-phase GSCs per niche was highest during the fourth larval stage (L4) and A1 (Fig. 1E). We conclude that GSC proliferative activity is highest during larval stages and steadily declines in unmated adult hermaphrodites, until it reaches a minimal level after 4 days spent in adulthood.

We wondered whether the GSC proliferative activity declined at the expense of increased differentiation. To test this, we induced differentiation in A1 or A4 equivalent animals (see Materials and Methods), using a conditional loss-of-function allele in the Notch receptor $(g l p-1)$. We found that inactivation of $g l p-1$ at A1 caused the differentiation of all GSCs in every observed germ line. However, inactivating $g l p-1$ at A4 resulted in $65.5 \%$ of animals having GSCs that remained undifferentiated (Fig. 1F-H). Thus, GSC proliferation does not decline at the expense of increased differentiation, and instead, both activities decline during adulthood in unmated hermaphrodites.

\section{Sperm depletion promotes GSC quiescence}

The decline in stem cell activities that we measured could result either from the deleterious effects of ageing (senescence), and/or from the promotion of quiescence. Quiescence is distinguished from senescence by the reversible nature of the cell cycle arrest or deceleration. Biomarkers of ageing consist of measurable activities that decay with age, and the kinetics of their decay reflects the decline in the population's survival (Fig. S2) (Johnson, 2006; Pickett et al., 2013). We compared the decline in GSC proliferation with the decline in survival of these animals. In unmated hermaphrodites, this revealed a bi-phasic decline curve of the GSC MI, with a rapid early decline, followed by stabilization. Interestingly, this curve did not obviously parallel the animals' survival decline (Fig. 1J). This suggests that ageing is not fully responsible for the GSC proliferative decline and that GSCs enter a nearly quiescent, slow-cycling state from A4 in unmated hermaphrodites.

Because the final decrease in GSC proliferative activity follows sperm depletion and the cessation of egg laying in unmated hermaphrodites (Byerly et al., 1976) (also see Fig. 2B), we hypothesized that sperm depletion promotes GSC quiescence. In this scenario, providing sperm to otherwise sperm-depleted hermaphrodites should increase their GSC MI. We therefore continually mated hermaphrodites with males to maintain sperm availability and investigated whether this would delay the GSC MI decline. We found that the GSC MI in continually mated A4 and A5 animals, following self-sperm depletion, remained significantly higher than in unmated animals (Fig. 1B,D,E). Likewise, we found that the GSC MI is drastically reduced already at A1 in fog-2 mutant hermaphrodites that cannot produce sperm, an effect that is completely reversed if these animals are mated (Fig. 1I). Together with previous observations in fog- 1 mutants (Morgan et al., 2010), these results indicate that sperm depletion inhibits GSC proliferation in adult hermaphrodites. We thus investigated whether the decline in GSC differentiation we observed in older (A4) unmated, animals 

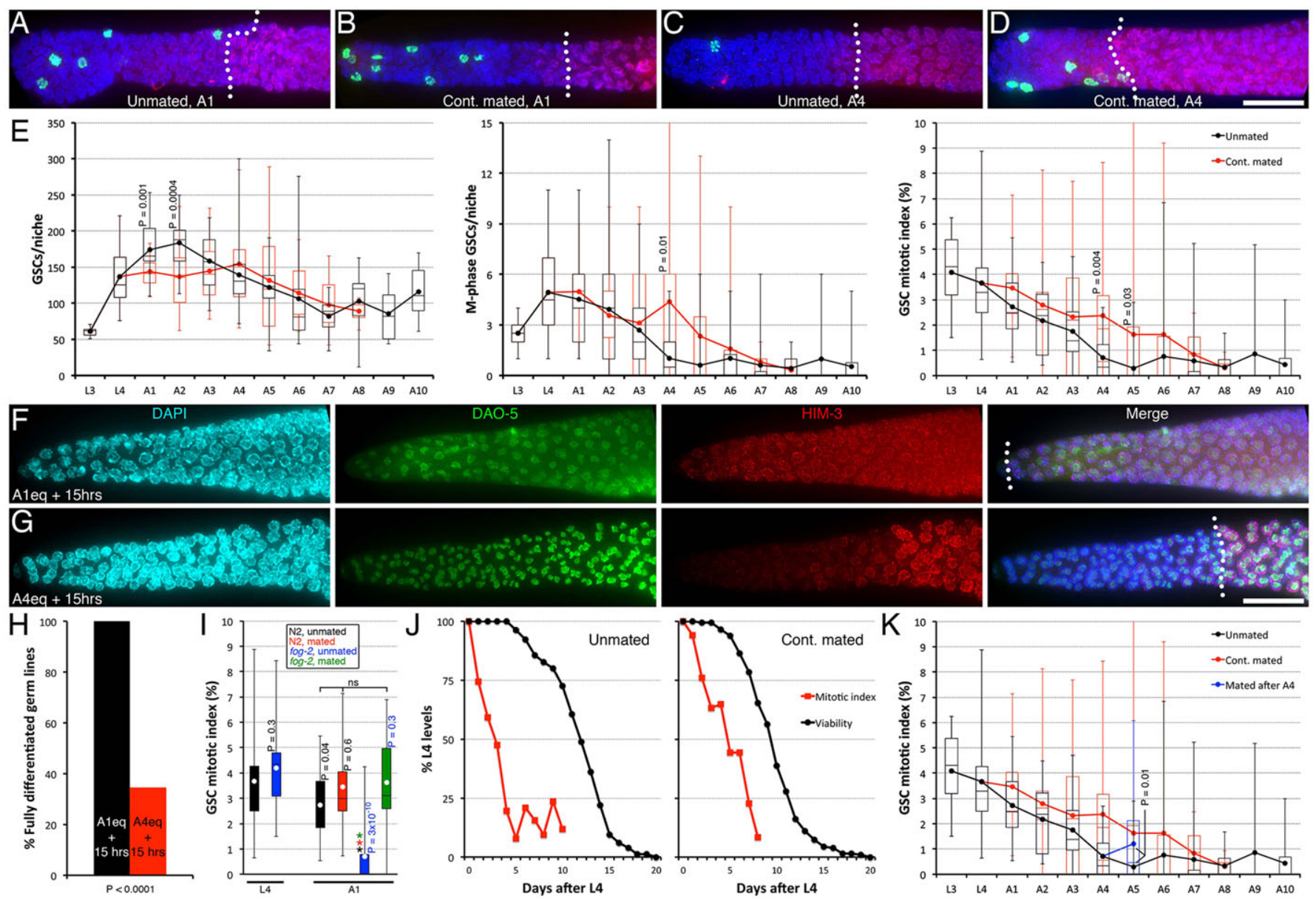

Fig. 1. Reversible promotion of GSC quiescence. (A-D) A representative distal germ line dissected from unmated $A 1$ ( $A$ ), continually mated $A 1$ (B), unmated $A 4$ (C) and continually mated A4 (D) wild-type animals, stained with DAPI (DNA; blue), anti-phospho[ser10] histone H3 (M-phase GSCs; green) and anti-HIM-3 (differentiated GSC progeny; red). (E) Time-course quantification of GSC numbers (left), M-phase GSCs (middle), and mitotic index (MI) (right) in unmated (black) and continually mated (cont. mated; red) wild-type hermaphrodites. (F-H) L4 hermaphrodites carrying a conditional loss-of-function mutation in the Notch receptor, glp-1(e2141), were maintained unmated under permissive conditions for the equivalent of 1 day (A1 eq; F) or 4 days (A4 eq; G) (see Materials and

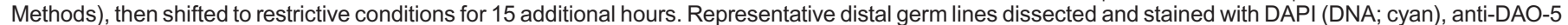
(nucleolar marker, used as a permeabilization control; green) and anti-HIM-3 (differentiation marker; red) are shown. $\mathrm{n}$, 16-29. Similar results were obtained with

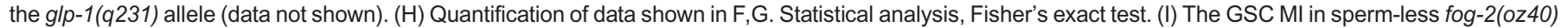
mutant hermaphrodites was normal during $L 4$, but was significantly reduced at A1. This was fully rescued if fog-2 hermaphrodites were mated. Colour-coded

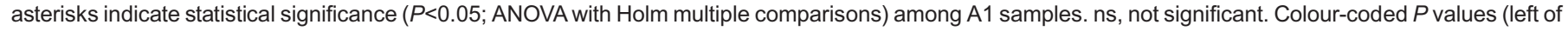

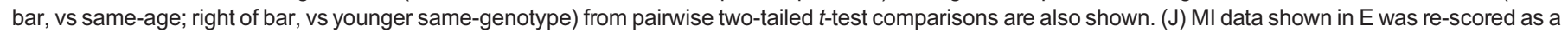

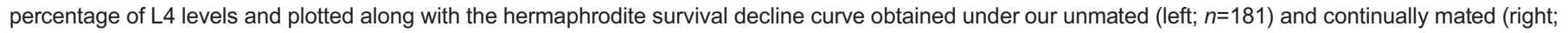

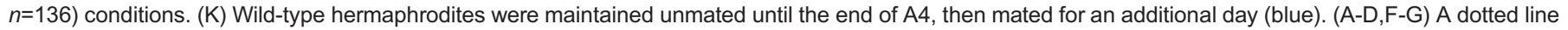

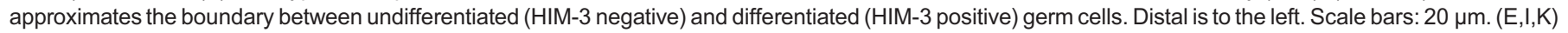

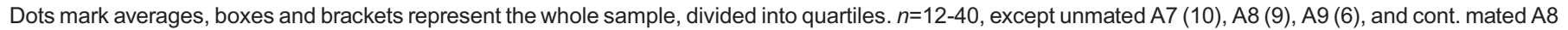
(3). (E,K) Significant $P$ values from pairwise two-tailed $t$-test comparisons are shown.

(Fig. 1F-H) could result from the fact that they were sperm depleted. Consistent with this, we found that GSCs differentiated less efficiently in A1 unmated fog-2 mutants than in their mated counterparts (Fig. S3). Thus, we conclude that sperm depletion promotes GSC quiescence, blocking both GSC proliferation and differentiation.

To verify that GSCs enter a state analogous to reversible quiescence in older, sperm-depleted hermaphrodites, we mated A4 hermaphrodites with males for an additional day to resupply them with sperm and thereafter measured their GSC MI. The GSC MI significantly increased in A5 animals that had been resupplied with sperm (Fig. 1K). We conclude that sperm depletion in adult hermaphrodites triggers a signal that promotes reversible GSC quiescence, regardless of the animal's age.
Of note, the GSC MI still declined with age in continually mated hermaphrodites (Fig. 1E). The GSC MI decline, however, roughly paralleled the survival decline under these conditions (Fig. 1J), which is compatible with the notion that it is a consequence of ageing (thus related to senescence), and that the GSC MI might be an ageing biomarker in mated hermaphrodites. Altogether, we conclude that the decline in GSC activities in ageing C. elegans adults is underlined by two mechanisms: a sperm depletion-sensing feedback that promotes reversible quiescence, combined with a sperm-independent mechanism that is potentially linked to ageing.

\section{Oocyte accumulation promotes GSC quiescence}

In animals lacking sperm (either by natural depletion or in fog mutants), oocyte maturation is inhibited and unfertilized oocytes 

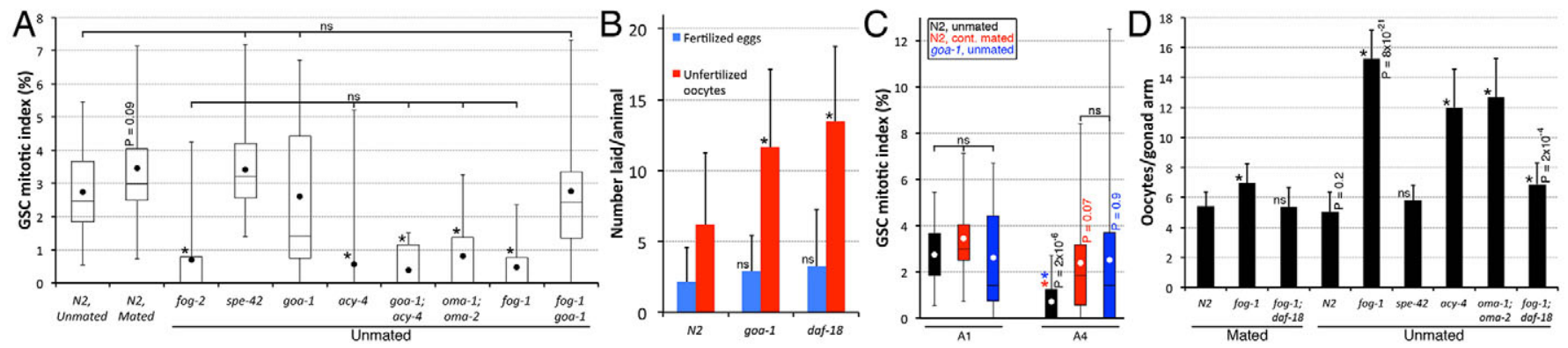

Fig. 2. Sperm depletion promotes GSC quiescence by blocking oocyte maturation and causing oocyte accumulation. (A) Oocyte maturation arrest promotes GSC quiescence downstream of MSP signalling. GSC MI was evaluated at A1 in unmated or mated animals of the indicated genotypes. ${ }^{*} P<0.05$ (ANOVA with Holm multiple comparisons) amongst unmated samples, versus unmated wild type. ns, not significant. The $P$ value is from a pairwise two-tailed $t$-test comparison versus unmated wild type. $n=15-25$. (B) goa-1 and daf-18 mutants lay significantly more unfertilized oocytes after sperm depletion. Animals were grown at $15^{\circ} \mathrm{C}$ until the mid-L4 stage, upshifted to $25^{\circ} \mathrm{C}$ for 3 days, after which they were singled on a new plate for $15 \mathrm{~h}$ at $25^{\circ} \mathrm{C}$. The number of fertilized eggs and unfertilized oocytes found on the plate after that 15-h period was recorded. ${ }^{*} P<0.05$ (ANOVA with Holm pairwise comparisons to control). ns, not significant. $n=19-20$ animals. (C) The GSC MI remains elevated in unmated, sperm-depleted, A4 goa-1(n1134) hermaphrodites, as in continually mated wild-type animals. Colour-coded asterisks indicate statistical significance $(P<0.05$; ANOVA with Holm multiple comparisons) among A1 or A4 samples. ns, not significant. Colour-coded $P$ values (vs younger same-genotype) from pairwise two-tailed $t$-test comparisons are also shown. $n=15-25$. (D) Oocyte accumulation levels of unmated or mated A1 animals of the indicated genotypes. Animals having endomitotic oocytes in the proximal gonad arm were excluded from this analysis. ${ }^{*} P<0.05$ (ANOVA with Holm pairwise comparisons to control). ns, not significant. $P$ values from pairwise two-tailed $t$-test comparisons versus mated animals of the same genotype are shown. $n=16-26$. (A-D) Alleles: fog-1(q785), fog-2(oz40), spe-42(tn1231), goa-1(n1134), acy-4(ok1806), oma-1(zu405te33), oma-2(te51), daf-18(nr2037). (A,C) Dots mark averages, boxes and brackets represent the whole sample, divided into quartiles. (B,D) Error bars represent s.d.

accumulate in each proximal gonad arm (Govindan et al., 2009; Lee et al., 2007; Miller et al., 2001). This raised the possibility that the accumulation of oocytes in the proximal gonad, as opposed to the lack of sperm, promotes GSC quiescence. To test this, we first asked whether oocyte fertilization is required to sustain high GSC proliferation rates. We found that the GSC MI was normal in spe42 mutant hermaphrodites (Fig. 2A), which produce fertilizationdefective sperm (Kroft et al., 2005), indicating that oocyte fertilization per se is not required to sustain elevated adult GSC proliferation rates.

Sperm, even in spe-42 mutants, secrete MSPs that are sensed by the proximal sheath cells of the somatic gonad and promote oocyte maturation and ovulation (Miller et al., 2001). To test whether MSP signalling is required to sustain high GSC proliferation rates, we evaluated the GSC MI in mutants with constitutive (goa-1) or defective (acy-4) MSP signalling. goa-1 encodes a G $\alpha$ subunit and mutants have constitutive MSP signalling and oocyte maturation (Govindan et al., 2006). Indeed, these animals did not retain oocytes after sperm depletion (Fig. 2B). We found that the GSC MI was normal in A1 goa-1 mutants, but remained significantly elevated after sperm depletion in A4 animals, with levels similar to wild-type animals that have unlimited sperm as a result of mating (Fig. 2A,C). Similarly, the GSC MI was elevated in A1 sperm-less fog-1 goa-1 double mutants (Fig. 2A). acy-4 encodes an adenylyl cyclase and mutants cannot relay MSP signalling, such that oocyte maturation and ovulation arrest, even in the presence of sperm (Govindan et al., 2009). We found that the GSC MI was low in A1 acy-4 mutants, with levels comparable to those of sperm-less fog mutants (Fig. 2A). Consistent with a previous analysis that placed $a c y-4$ downstream of goa-1 in MSP signalling (Govindan et al., 2009), acy-4 loss of function $[a c y-4(l f)]$ completely suppressed the elevated GSC MI phenotype of goa-1(lf) (Fig. 2A). These results indicate that GSC proliferation rates do not directly respond to the presence of sperm, but rather to an activity that depends on MSP signalling.

MSP signalling promotes oocyte maturation by positively regulating the activity of the redundant zinc finger proteins OMA1 and OMA-2 (Detwiler et al., 2001). To determine if oocyte maturation, downstream of MSP signals, is required to sustain high adult GSC proliferation rates, we measured the GSC MI in oma-1; oma-2 double mutants. The GSC MI of these animals was low at $\mathrm{A} 1$, as in sperm-less fog mutants (Fig. 2A). We conclude that an arrest in oocyte maturation promotes GSC quiescence.

We noted that in every case in which oocyte maturation was inhibited and GSC quiescence was promoted, there was a striking accumulation of seemingly compressed, unfertilized oocytes in the proximal gonad (Fig. 2D; Fig. S1B) (Govindan et al., 2009; Lee et al., 2007; Miller et al., 2001). We therefore investigated whether the presence of oocytes was necessary to induce GSC quiescence by assessing the GSC MI in animals that accumulated other germ cell types. We first measured the GSC MI in fem-3 gain-of-function $[\mathrm{fem}-3(\mathrm{gf})]$ mutant hermaphrodites that accumulate sperm (Barton et al., 1987). The GSC MI remained elevated in fem-3(gf) hermaphrodites even after sperm had dramatically accumulated (Fig. 3A,B). Furthermore, measuring the GSC MI in males, which only produce sperm, revealed that GSC proliferation rates remained elevated relatively longer than in unmated hermaphrodites, as in continually mated hermaphrodites (Fig. 3B,C). Thus, quiescence may not be promoted in the GSCs of males. Finally, a MAPK (mitogen-activated protein kinase, also known as ERK, called MPK-1 in C. elegans) weak reduction-of-function allele that dramatically inhibits pachytene progression (no oocytes are made in the majority of animals), allowed us to evaluate GSC proliferation rates when there is an accumulation of mid-pachytene stage germ cells (Lackner and Kim, 1998; Lee et al., 2007). We found that the GSC MI in these mutants did not rapidly decrease by A1 (such as in sperm-less fog mutants that accumulate oocytes) and remained indistinguishable from that of wild-type hermaphrodites, at least until A2 (Fig. 3D). Thus, an accumulation of sperm or pachytenearrested germ cells does not promote GSC quiescence. We conclude that a block in oocyte maturation and/or the resulting accumulation of oocytes, the terminally differentiated progeny of the stem cells, specifically feeds back to promote GSC quiescence.

\section{Oocyte accumulation promotes GSC quiescence beyond the niche}

In C. elegans mutants with hyperactive Notch signalling, GSCs can remain in the self-renewal state even when they move outside of the 

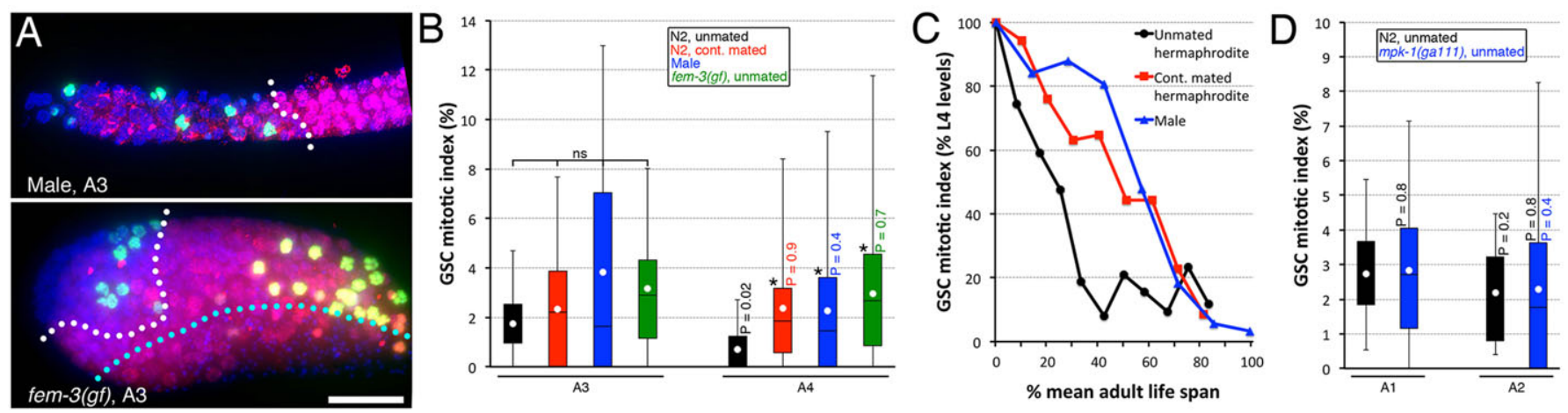

Fig. 3. Accumulation of other germ cell types does not promote GSC quiescence. (A) A3-4 fem-3(q20)gf hermaphrodites (bottom) have accumulated large amounts of sperm cells, such that it backs up [in 15\% of A3 ( $n=27)$ and $68 \%$ of A4 ( $n=47)$ animals] within 25 cell diameters of the distal tip. An A3 him-5(e1490) male (top) is shown as a control. DAPI (DNA; blue), anti-phospho[ser10] histone H3 (M-phase GSCs; green), anti-HIM-3 (differentiated GSC progeny; red). White dotted line indicates approximate boundary between undifferentiated (HIM-3 negative) and differentiated (HIM-3 positive) germ cells. Cyan dotted line indicates approximate distal sperm (small bright DAPI spots) boundary. Distal is to the left. Scale bar: $20 \mu m$. (B) Sperm accumulation in fem-3(gf) hermaphrodites does not promote GSC quiescence. GSC MI in A4 fem-3(q20)gf hermaphrodites is significantly higher than in unmated wild-type controls but is not different from that in continually mated wild-type hermaphrodites or in him-5(e1490) males. $n=13-34$, except for A3 males (9). (C) GSC MI decline, expressed as a percentage of L4 levels, in him-5(e1490) males obviously parallels better with the decline in continually mated wild-type hermaphrodites than that in unmated hermaphrodites when corrected for each condition's mean adult life span. $n=102-181$. (D) Although GSC quiescence is promoted before A1 in fog-1 or fog-2 mutants that accumulate oocytes (see Fig. $1 \mathrm{l}$ and Fig. 2A), GSC quiescence is still not promoted at A2 in a weak mpk-1 reduction-of-function mutant that causes an accumulation of pachytene-arrested germ cells (Leacock and Reinke, 2006; Lee et al., 2007). mpk-1(ga111) was linked to unc-79(e1068). $n=20-34$. (B,D) Dots mark averages, boxes and brackets represent the whole sample, divided in quartiles. ${ }^{*} P<0.05$ (ANOVA with Holm pairwise comparisons to control) among A3 or A4 samples. ns, not significant. Colour-coded $P$ values (left of bar, vs same-age control; right of bar, vs younger same-genotype/condition) from pairwise two-tailed $t$-test comparisons are also shown.

niche, and proliferate until they overtake the entire germ line (Fig. 4A,B; Fig. S1C) (Berry et al., 1997). These cells have thus been thought of as analogous to those in the early stages of a benign tumour in vertebrates (Pinkston et al., 2006). To gain insight into the type of signalling that is involved in feedback GSC regulation, we investigated whether oocyte accumulation could suppress the ectopic growth of tumorous germ cells and induce quiescence outside of the niche area. We tested this by assessing tumour growth, hence GSC proliferative activity, in fog- 1 mutants bearing a conditional $g f$ allele in the Notch receptor (Pepper et al., 2003). When these double mutant animals were grown under restrictive conditions from the first larval stage (L1), most developed a clearly visible germline tumour by A3-4, similar to $g l p-1(g f)$ single mutants (Fig. 4A-C). To test whether the presence of oocytes blocks tumour formation, we allowed these sperm-less fog- $1 ; \operatorname{llp}-1(g f)$ double mutants to grow under permissive conditions until gametogenesis had begun (L4 stage) and then shifted them to restrictive conditions. This allowed the production of oocytes and their accumulation in the proximal arm (due to the lack of sperm). Under these conditions, the vast majority of double mutant animals did not develop germline tumours (Fig. 4E). Similar results were obtained using fog-2 mutants (Fig. S4). This is in contrast to $g l p-1(g f)$ single mutants grown under a similar regime, which produced a few eggs and developed a germline tumour by A3-4 (Fig 4D). Activating MSP signalling and preventing oocyte accumulation by mutating $g o a-1$ in these $g l p-1(g f)$; fog- 1 mutants caused a reversion to the tumorous phenotype (Fig. 4F), suggesting that the presence of arrested oocytes is needed to block GSC proliferation in these animals. Together, these results indicate that the feedback signal generated when oocytes accumulate is not restricted to the distal tip and can block the growth of tumorous germ cells beyond the niche.

\section{Germline IIS levels control adult GSC proliferation rates}

Previous work has shown that the induction of GSC quiescence during the juvenile diapause-like dauer stage can be triggered by a downregulation of IIS (Narbonne and Roy, 2006b; Narbonne et al., 2010). Moreover, a reduction-of-function mutation in $d a f-2$, encoding the sole C. elegans insulin/IGF-1 receptor homologue (Kimura et al., 1997), suppresses GSC proliferation during larval development (Michaelson et al., 2010), protects against germline tumour growth during adulthood (Pinkston et al., 2006), and thus phenocopies oocyte accumulation (Fig. 4D,E,G). We therefore tested whether IIS was affected downstream of sperm depletion, to promote GSC quiescence. Previous analyses failed to observe an IIS requirement for adult GSC proliferation rates using the widely used temperaturesensitive daf-2(e1370) allele at a semi-permissive temperature of $20^{\circ} \mathrm{C}$ (Michaelson et al., 2010; Pinkston et al., 2006). Likewise, we found no significant effect of the daf-2(e1370) mutation on the GSC MI of adult animals at $20^{\circ} \mathrm{C}$ (Fig. 5A). We found that a stronger daf-2 inactivation, using either the $e 1370$ or $e 979$ alleles at the fully restrictive temperature of $25^{\circ} \mathrm{C}$, significantly lowered the adult GSC MI (Fig. 5B-D). Similarly, unmated and mated daf-2; fog-2 double mutants both had a reduced GSC MI (Fig. 5C). We conclude that DAF-2, the insulin/IGF-1 receptor, is required to sustain elevated GSC proliferation rates in adult hermaphrodites.

We next investigated whether $d a f-18$ was required downstream of daf-2 to promote GSC quiescence in response to sperm depletion. We first verified whether the inhibition of adult GSC proliferation and the suppression of $g l p-1(g f)$-induced germline tumours by daf-2 downregulation were dependent on daf-18. Indeed, double daf-2; daf-18 mutants had a normal MI (Fig. 5C), and triple daf-2 $g l p-1(g f)$; daf-18 mutants rapidly developed germline tumours, similar to $g l p-1(g f)$ single mutants (Fig. 4G,H). Thus, daf-2 inactivation requires $d a f-18$ to promote GSC quiescence. We next investigated whether sperm depletion similarly required $d a f-18$ to promote GSC quiescence. We found that mutating daf-18 in fog-1; $g l p-1(g f)$ animals reverted the germline tumour suppression (Fig. 4I). Furthermore, the GSC MI was not downregulated in A1 sperm-less fog-1; daf-18 double mutants, and remained elevated in A4 sperm-depleted daf-18 single mutants, such that animals that lacked daf-18 were indistinguishable from continually mated 


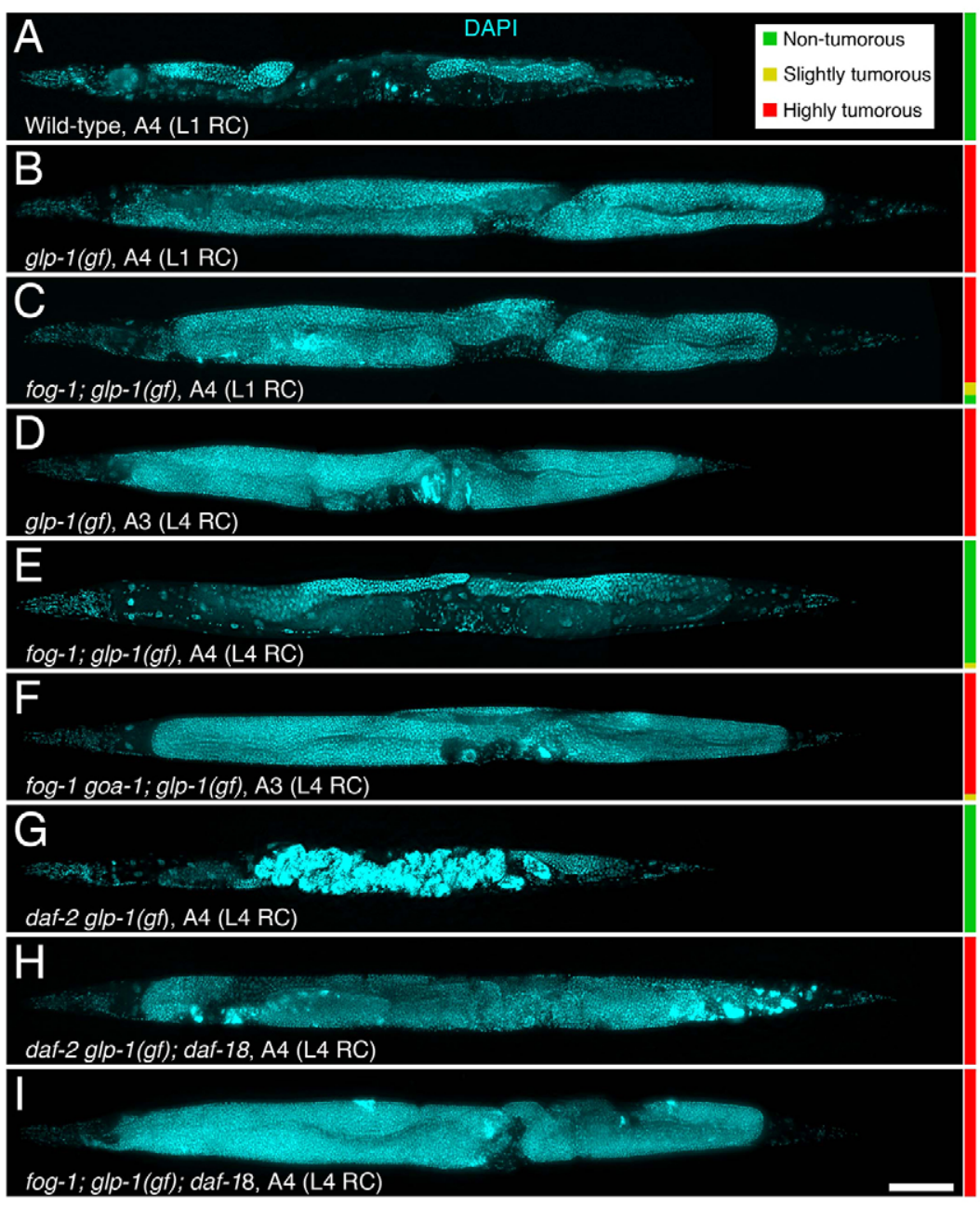

Fig. 4. Oocyte accumulation, similar to IIS downregulation, blocks tumorous germ cell proliferation. (A-I) Representative DAPI-stained (cyan) A3 to A4 animals of the indicated genotype, either grown under restrictive conditions (RC) from the L1 stage (top three panels), or grown under permissive conditions until the mid-L4 stage, then shifted to RC thereafter (bottom six panels) are shown. Alleles: conditional glp-1(ar202)gf, fog-1(q785), conditional daf-2(e1370), daf-18(nr2037), goa-1(n1134). Anterior is to the left and dorsal, to the top. A colour-coded quantification of the phenotype is shown on the right, scored at A4 (animals were scored as highly tumorous if more than one-third of the animal was translucid, slightly tumorous if between one-fifth and onethird was translucid, and non-tumorous if less than one-fifth of the animal was translucid; the relative lengths of the colourcoded bars represent percentages). Although $g / p-1$ (gf) animals are clearly tumorous already by A1-2 when grown under restrictive conditions (Pepper et al., 2003), animals are shown here at A3-4 to show that oocyte accumulation suppresses ectopic GSC proliferation for a prolonged duration. $n=28-52$. Scale bar: $100 \mu \mathrm{m}$.

hermaphrodites (Fig. 5E,F). Sperm depletion therefore requires daf-18 to promote GSC quiescence. Consistent with sperm depletion acting sequentially with $d a f-18$, continually mating $d a f-$ 18 mutants did not further influence GSC proliferative activity (Fig. 5F). We noticed that oocytes significantly accumulated in A1 sperm-less fog-1; daf-18 double mutants, but not to the level of fog- 1 single mutants (Fig. 2D), and that A4 sperm-depleted daf-18 single mutants were defective in oocyte retention, similar to goa-1 mutants (Fig. 2B). Thus, daf-18 mutants closely resemble goa-1 mutants, which have constitutive MSP signalling. We conclude that sperm depletion blocks MSP signalling, which then requires daf-18 to fully arrest oocyte maturation/ovulation and promote GSC quiescence.

We then wondered if daf-16 was required to promote GSC quiescence in response to IIS receptor inactivation and sperm depletion. Like $d a f-18$, we found that $d a f-16$ was required for $d a f-2$ inactivation to inhibit adult GSC proliferation (Fig. 5C). Surprisingly, however, unlike in daf-18 mutants, the GSC MI in daf-16 null mutants normally decreased by A4, following sperm depletion (Fig. 5F). Moreover, the GSC MI was low in A1 spermless daf-16; fog-2 double mutants, as in fog-2 single mutants (Fig. 5G). Consistent with this, oocytes accumulate normally in the proximal gonad of daf-16; fog-2 mutants (Qin and Hubbard, 2015), indicating that $d a f-16$ is not required for oocyte accumulation, unlike $d a f-18$. We conclude that upon sperm depletion, daf-18 is required to promote oocyte accumulation and GSC quiescence, independently of daf-16.
IIS could act cell-autonomously in the germ line to sustain elevated GSC proliferation rates. Consistent with this, it was previously shown that germline-specific inhibition of $d a f-2$ is sufficient to inhibit GSC proliferation, requiring germline daf-16 (Michaelson et al., 2010). Somatic daf-2 inactivation, however, nonautonomously affects many processes (Apfeld and Kenyon, 1998) and could thus also potentially influence GSC proliferation rates. We therefore investigated whether soma-specific daf-2 inhibition could block GSC proliferation. Germline-specific daf-2 expression, however, restored a high GSC MI in daf-2 mutants (Fig. 5C), indicating that soma-specific daf-2 inactivation is not sufficient to block GSC proliferation, at least in well-fed animals. Therefore, when animals are maintained on food, canonical IIS is activated in the germ line, and this is both necessary and sufficient to promote elevated GSC proliferation rates. We conclude that germline IIS needs to be antagonized by other signals downstream of sperm depletion, requiring $d a f-18$, but not $d a f-16$, in order to promote GSC quiescence in well-fed animals.

\section{Oocyte accumulation promotes GSC quiescence independently in each gonad arm}

The feedback signal linking sperm availability to oocyte maturation was shown to affect each gonad arm independently (Govindan et al., 2009). IIS, however, tends to be systemically aligned throughout tissues owing to the secreted and diffusible nature of its ligands, as well as signalling between tissues (Murphy and $\mathrm{Hu}, 2013$; Murphy et al., 2007). We therefore wondered whether the daf-18-dependent 

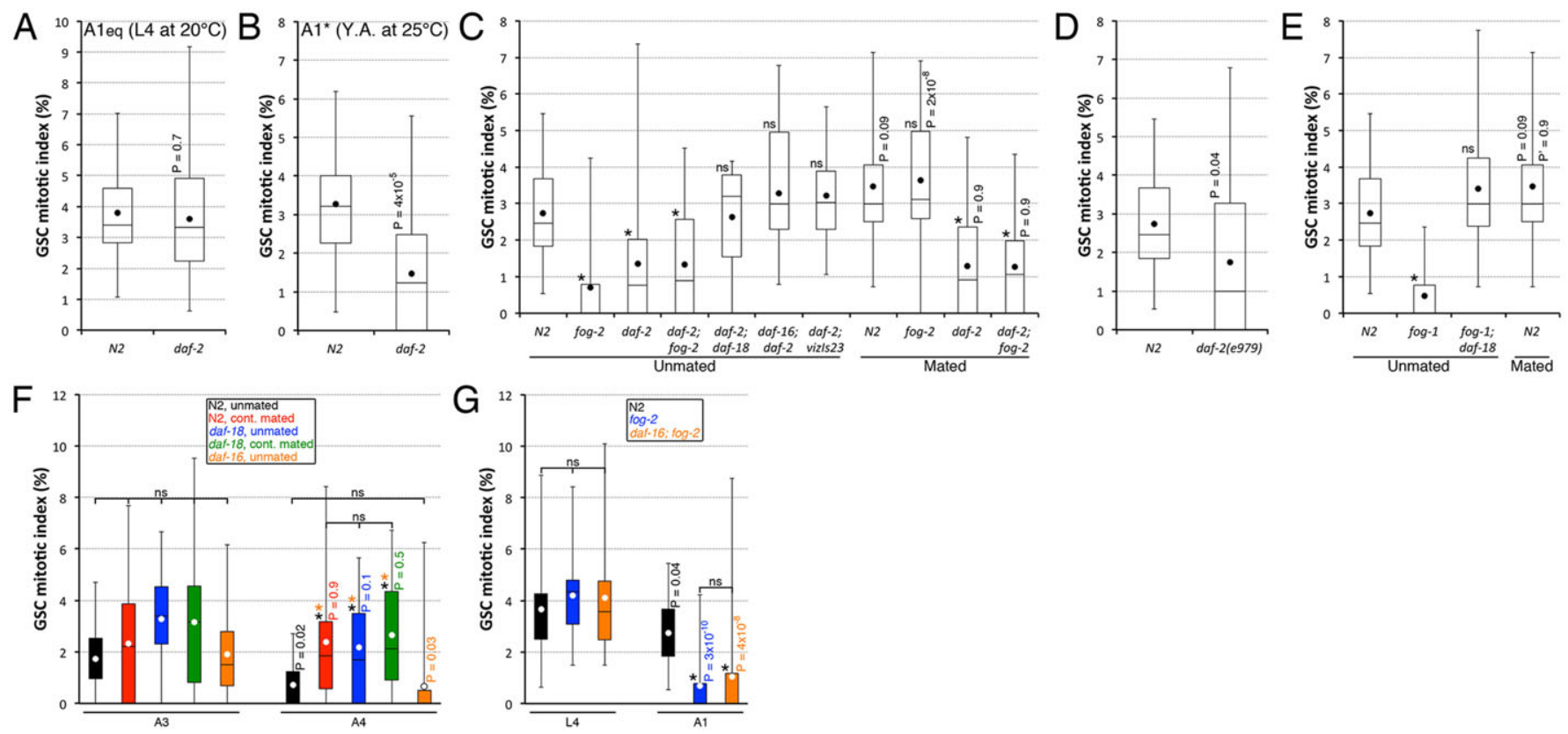

Fig. 5. Sperm depletion requires daf-18 to antagonize germline IIS and promote GSC quiescence. (A,B) Wild-type or daf-2(e1370) animals were either grown at $15^{\circ} \mathrm{C}$ until the $L 4$ stage and upshifted to $20^{\circ} \mathrm{C}$ for $36 \mathrm{~h}(\mathrm{~A})$, or grown at $15^{\circ} \mathrm{C}$ until the young adult (Y.A.) stage and upshifted to $25^{\circ} \mathrm{C}$ for $24 \mathrm{~h}(\mathrm{~B})$, before their $\mathrm{GSC}$ MI was scored. $n=23-31$. (C-E) GSC Ml comparison of unmated or mated A1 hermaphrodites of the indicated genotypes. ${ }^{*} P<0.05$ (ANOVA with Holm pairwise comparisons to the relevant unmated/mated wild-type control). ns, not significant. $P$ values from pairwise two-tailed $t$-test comparisons versus unmated animals of the same genotype (C,E), or versus the wild-type control (D), are shown. (C) Alleles: fog-2(oz40), daf-2(e1370), vizls23[pie-1p::GFP::DAF-2(+); unc-119(+)] (Lopez et al., 2013), daf-18(nr2037), daf-16(mu86). The daf-2; daf-18 and daf-16; daf-2 strains also carry ojls1. $n=16-35$. (D) $n=25-28$. (E) Alleles: fog-1(q785), daf18(nr2037). The $P$ value from the pairwise two-tailed $t$-test comparison versus unmated fog-1; daf-18 $\left(P^{\prime}\right)$ is also shown. $n=23-32$. (F) GSC quiescence is not promoted by sperm depletion in unmated daf-18(nr2037) null hermaphrodites, but it is promoted normally in sperm-depleted A4 daf-16(mgDf50) null mutants. GSC $\mathrm{MI}$ decline is similar in continually mated and unmated A3 and A4 daf-18 hermaphrodites. $n=13-30$. (G) GSC quiescence is normally promoted in unmated A1 daf16(mgDf47); fog-2(q71) mutant hermaphrodites, as in fog-2(oz40) single mutants. $n=23-38$. (A-G) Dots mark averages, boxes and brackets represent the whole sample, divided in quartiles. $(\mathrm{F}, \mathrm{G})$ Colour-coded asterisks indicate statistical significance $(P<0.05$; ANOVA with Holm multiple comparisons) among L4, A1, A3 or A4 samples. ns, not significant. Colour-coded $P$ values (vs younger same-genotype/condition) from pairwise two-tailed $t$-test comparisons are also shown.

feedback regulation of GSC proliferative activity acts independently in each gonad arm. To test this, we micro-injected an oil diaphragm specifically into the anterior or posterior spermatheca and/or uterus of young adult $f o g-1 ; g l p-1(g f)$ mutants that had been grown under permissive conditions, in order to allow oocyte accumulation and the induction of GSC quiescence (as in Fig. 4E). These animals were then shifted to restrictive conditions and allowed to mate for $3 \mathrm{~h}$ with males. The oil diaphragm acted as contraception and blocked male sperm from accessing the accumulated oocytes in the injected arm, allowing sperm to activate and fertilize oocytes in the untreated arm of the animal (Fig. S1D). We found that preventing sperm access (either in the anterior or posterior arm) with an oil diaphragm specifically protected this gonad arm from developing a tumour (Fig. 6A-C,F). Oil diaphragm injection required oocytes to inhibit GSC proliferation because it did not block the growth of germline tumours in gld-2; gld-1 double mutants (Fig. 6D-F), which do not generate any gametes (Kadyk and Kimble, 1998). Interestingly, in all cases, the proximal-most oocytes in the oil-injected arm underwent activation, probably owing to the diffusion of sperm-secreted MSPs around the oil diaphragm and into the proximal gonad (Govindan et al., 2009; Miller et al., 2001). However, because sperm remained blocked in the uterus, these activated oocytes became endomitotic (Iwasaki et al., 1996) (Fig. 6B,C). This indicates that oil injection per se did not compromise oocyte function. Also, in every case where the induction of GSC quiescence was maintained, a few of the distalmost oocytes remained arrested (Fig. $6 \mathrm{~B}^{\prime}, \mathrm{C}^{\prime}$ ). This result suggests that the trigger for promoting GSC quiescence might not be directly related to the actual number of arrested oocytes. As oocyte accumulation in one gonad arm promotes GSC quiescence specifically in that arm, we conclude that when oocytes accumulate, IIS is antagonized in a gonad arm-specific manner.

To verify that oocyte accumulation does not affect IIS across animals, we monitored the subcellular localization of GFP::DAF-16 in fog-2 mutants, as a readout of systemic IIS. In well-fed control A1 animals, GFP::DAF-16 was predominantly cytoplasmic owing to a systemic activation of IIS (Fig. 6G) (Lee et al., 2001; Lin et al., 2001). GFP::DAF-16 remained predominantly cytoplasmic following oocyte accumulation in well-fed fog-2 mutants, supporting the notion that IIS is not systemically inhibited in these animals (Fig. 6G). As expected, a $3 \mathrm{~h}$ starvation period rapidly triggered systemic GFP::DAF-16 nuclear translocation in fog-2 mutants, indicating that systemic DAF-16 activation is not impaired in these mutants (Fig. 6G). It was also reported that germline feminization does not extend C. elegans life span (Arantes-Oliveira, 2002), which would have been expected if oocyte accumulation caused a systemic downregulation of IIS. Therefore, we conclude that when oocytes accumulate following sperm depletion, IIS is locally antagonized to promote GSC quiescence specifically in the affected gonad arm, and it is not otherwise perturbed in the rest of the animal.

\section{DISCUSSION}

Our time-course analyses of GSC proliferation decline in ageing C. elegans adults highlighted two underlying mechanisms: a passive decline that is probably linked to ageing, and a feedback 

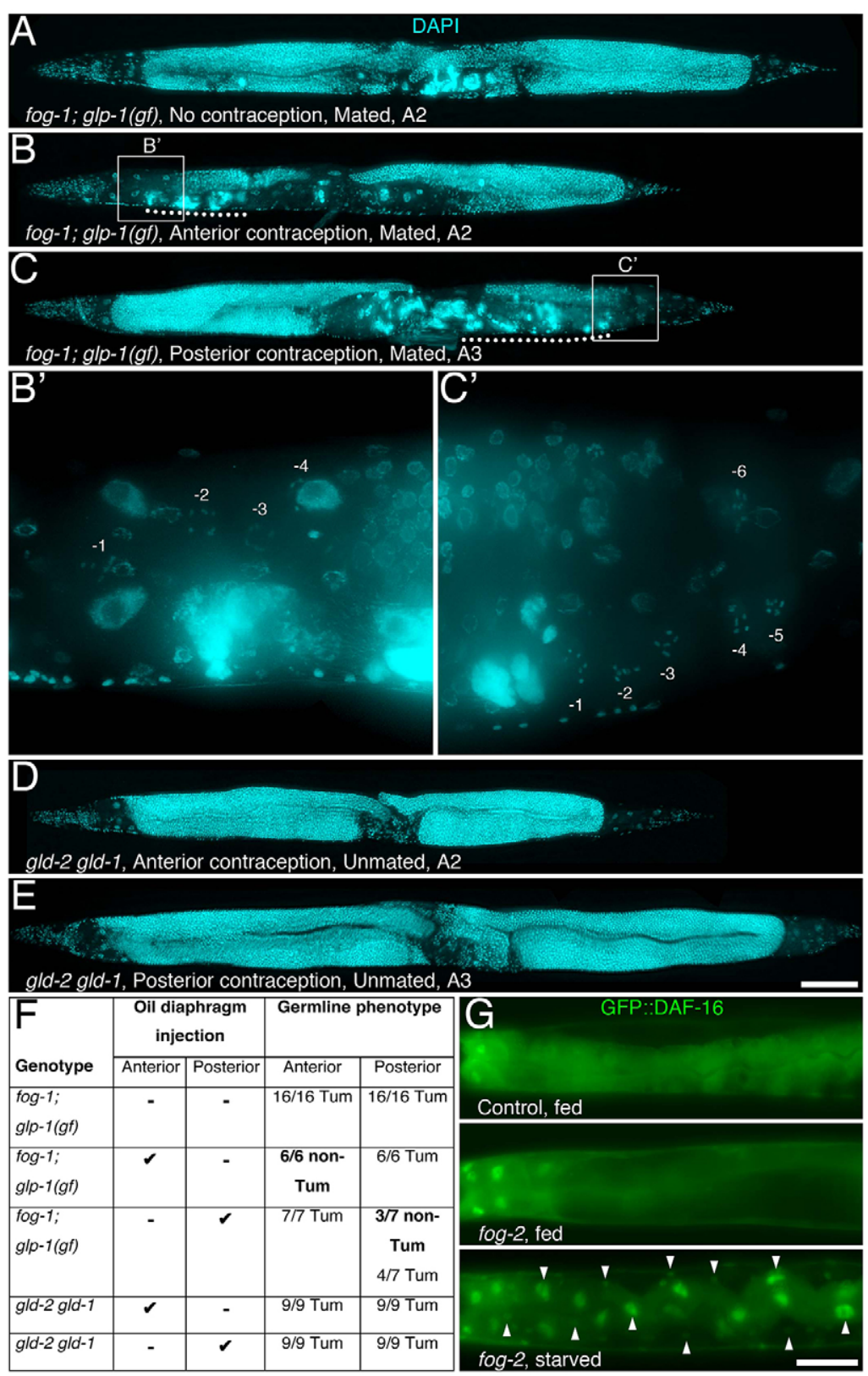

Fig. 6. Independent regulation of GSC proliferation, and thereby of IIS outputs, in each gonad arm. (A-E) An oil diaphragm was micro-injected into the anterior or posterior spermatheca and/or uterus of fog-1(q785); glp-1(ar202)gf young adult hermaphrodites that had been grown under permissive conditions. Animals were then shifted to restrictive conditions and allowed to mate with males for $3 \mathrm{~h}$ (Fig. S1D). The germline phenotype was scored 2-4 days after injection in animals that successfully mated (based on progeny production). Oil was also micro-injected in the anterior or posterior spermatheca and/or uterus of $g / d-2(q 497)$ gld-1(q485) young adults (these were not mated), which are non-conditional tumorous (Hansen and Schedl, 2013) and do not accumulate oocytes, to verify that the effect of the oil diaphragm was dependent on the presence of oocytes. A representative DAPI-stained animal is shown for each condition. Intact proximal-most oocytes are labelled with negative values from the proximal end in close-ups. Activated, but not fertilized, endomitotic oocytes are underlined by a dotted line. $(A-C, D, E)$ Scale bar: $100 \mu \mathrm{m}$. (F) Summary of contraception experiments. Tum, tumorous. (G) A functional GFP.:DAF-16 protein (Murphy et al., 2007) is predominantly cytoplasmic in wellfed A1 controls (top, 100\%), as well as in A1 unmated fog-2 (oz40) (middle, 96\%) hermaphrodites, which have quiescent GSCs. Systemic IIS inactivation is not impaired in fog-2 mutants, as a $3 \mathrm{~h}$-starvation period caused a rapid systemic nuclear enrichment of GFP::DAF-16 (bottom, 92\%) in A1 fog-2 mutants. Similar results were observed in fog-1(q785) animals (data not shown). A representative animal is shown for each condition, cropped posterior to the pharynx, anterior to the left and dorsal up. Arrowheads point at some of the cells with obvious nuclear GFP::DAF-16 enrichment. $n=24-41$. Scale bar: $50 \mu \mathrm{m}$. process that can promote quiescence. By experimentally separating these two phenomena, we discovered that it is an accumulation of oocytes, a terminally differentiated progeny of the GSCs, which triggers a signal that promotes GSC quiescence. We found that each gonad arm independently responds to this signal, and that it affects GSCs beyond the niche area, probably throughout the affected gonad arm. Finally, we demonstrated that sperm depletion requires daf-18, but not daf-16, to antagonize germline-intrinsic canonical IIS to promote GSC quiescence. Together, these results support a model in which canonical IIS levels are systemically set by nutrient intake and dictate the maximal GSC proliferation rate at a given age. Maintenance of the maximal GSC proliferation rate in adults, however, requires the continuous stimulation of oocyte maturation by sperm-secreted MSPs, such that when oocyte maturation is arrested and oocytes accumulate in the proximal gonad, systemic IIS information is locally antagonized in that gonad arm to promote GSC quiescence (Fig. 7). Thus, in multicellular organisms, nutritional systemic information that would otherwise equally influence every cell can be locally overridden by other factors to promote stem cell quiescence when proliferation and differentiation is temporarily not needed.

\section{Control of GSC proliferation rates by germline IIS neutralization}

We found that canonical IIS needs to be blocked within the germ line, requiring daf-18 and daf-16 activity, in order for the GSCs to slow down their division rate and approach quiescence when sperm is available and nutrient uptake is optimal. daf-16 was, however, dispensable for oocyte accumulation and the promotion of GSC quiescence in sperm-depleted animals. The establishment of GSC quiescence during dauer development similarly requires $d a f-18$ and daf-16 if dauer formation is induced by IIS downregulation, but only daf-18 if it is induced by another (TGF $\beta$ ) signalling pathway (Narbonne and Roy, 2006b). Interestingly, the TGF $\beta$ pathway has been recently implicated in the regulation of reproductive ageing (Luo et al., 2010) and GSC proliferation (Dalfó et al., 2012) in 
Worm on food: systemic insulin signalling activation

Anterior arm: $\quad$ Posterior arm:

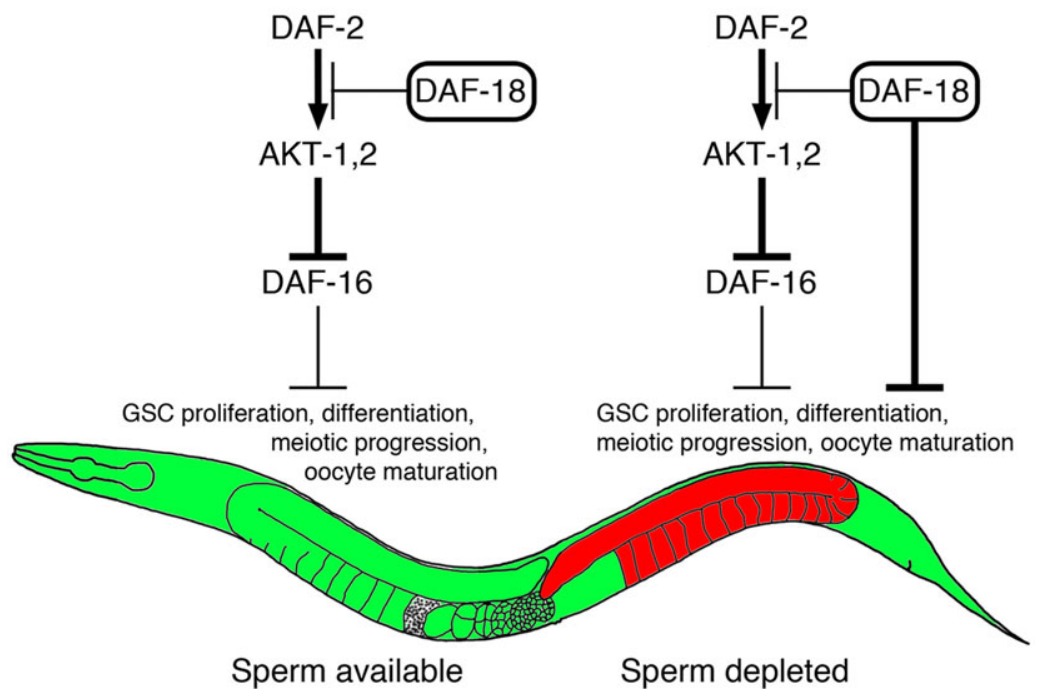

Fig. 7. Model for localized IIS antagonism downstream of sperm depletion. When C. elegans adult hermaphrodites are grown under abundant food conditions, IIS is systemically activated (green). If, in a given gonad arm, sperm is available, oocytes never accumulate as they are continually fertilized. This leaves canonical IIS (daf-2 to daf-16) unchecked throughout that gonad arm, or at its highest level as governed by nutrient status, and able to simultaneously promote GSC proliferation, differentiation, progression through meiosis (Lopez et al., 2013), and oocyte maturation. However, if a gonad arm becomes sperm deleted, oocyte maturation is inhibited, causing their accumulation, which then promotes GSC quiescence locally within that gonad arm. We found that daf-18 is required to locally antagonize germline IIS (red) and block oocyte maturation and GSC proliferation in that gonad arm in response to sperm depletion, thus locally antagonizing the systemic IIS information about nutrient status. Because daf-18 mutants are partially defective in oocyte accumulation in response to sperm depletion, it remains unclear whether daf-18 directly inhibits GSC proliferation in response to sperm depletion, and/or whether it is working indirectly by blocking oocyte maturation and causing their accumulation. Also, because daf-16 is not required for sperm depletion to block oocyte maturation and GSC proliferation, a model in which daf-18 is acting in parallel to the canonical IIS is favoured in this context. An arrow indicates stimulation, a bar indicates inhibition, and thickness represents relative activity level.

C. elegans. Whether oocyte accumulation somehow impinges on TGF $\beta$ signalling to locally antagonize IIS to promote GSC quiescence is thus an interesting possibility that will require further investigation.

Nutrient uptake systemically regulates IIS levels, and thereby impinges on stem cell proliferation rates in most multicellular organisms (LaFever and Drummond-Barbosa, 2005; Shim et al., 2013). However, stem cell populations do not always proliferate at an equal rate within an individual. Indeed, at a given moment, stem cell proliferation rates can differ across different tissues (Tomasetti and Vogelstein, 2015), or even within parts of the same tissue (this study). We found that the differential negative feedback regulation of two GSC populations within the germ line of well-nourished C. elegans animals happens through localized neutralization of an output (stimulation of GSC proliferation) from the otherwise systemically controlled IIS (Fig. 7). This concept might underlie many other feedback-regulated stem cell systems. Elucidating how such local regulation of systemic signal outputs is accomplished could contribute to the development of targeted, tissue-specific treatments for metabolic and proliferative diseases, including cancer.

\section{Control of adult stem cell pool size versus proliferation rate} It was recently reported that the GSC pool size is regulated non-cellautonomously in ageing $C$. elegans adults by daf-16 activity in the proximal somatic gonad (Qin and Hubbard, 2015). In the present study, we did not focus our attention to the GSC pool size (which reports on a combination of prior expansion, ongoing proliferation and exit from the pool due to differentiation) but rather on the GSC MI, which is an indication of the level of GSC proliferative activity.
Despite multiple differences in experimental design, including the temperature, both studies find that the GSC pool size declines in ageing hermaphrodites. Our results, however, add the notion that the GSC proliferative activity is not necessarily related to the pool size. Indeed, the time during which the GSCs are the most mitotically active (L3) corresponds to a time when the pool size is relatively small. Conversely, the time when the GSC pool size is maximal (A2) does not correspond to a peak in the MI. Thus, the GSC pool size and GSC proliferation rates are two distinct parameters that are regulated independently. The GSC pool size in ageing $C$. elegans adults is influenced by daf-16-dependent factors in the proximal somatic gonad (Qin and Hubbard, 2015), potentially through affecting the size of the niche cell (DTC) and/or the extent of niche signalling. Although IIS levels are systemically set by nutrient uptake, our work indicates that its final germline-intrinsic outputs that control GSC proliferation rates can be antagonized by oocyte needs locally in each gonad arm.

\section{Is oocyte accumulation the trigger for GSC quiescence?}

None of the mutant backgrounds that we analysed allowed a significant uncoupling between oocyte maturation, oocyte accumulation and GSC proliferation. That is, either oocyte maturation was inhibited, in which case oocytes accumulated in the proximal gonad and GSCs approached quiescence, or oocyte maturation proceeded normally, in which case oocytes did not accumulate and GSC proliferation was elevated. Therefore, it is currently unclear whether it is the arrest in oocyte maturation or the resulting accumulation of oocytes that feeds back to promote GSC quiescence. The coupling between oocyte accumulation and GSC 
proliferation might result from the requirement in daf-18 activity for both oocyte and, directly or indirectly, GSC quiescence (Fig. 7). Indeed, we found that oocytes spontaneously activate and are ovulated in sperm-depleted daf-18 null mutants, much like in goa-1 mutants with hyperactive MSP signalling. In this view, the C. elegans germ line would behave as an assembly unit, with IIS being the ON/OFF switch that simultaneously acts at the levels of GSC proliferation, differentiation, meiotic progression (Lopez et al., 2013) and oocyte maturation, to promote mature oocyte production at a fast pace. However, oocytes keep maturing and accumulate for some time in the absence of sperm, or after sperm is depleted, indicating that the two events can be temporally uncoupled, at least for a moment. Consistent with this, pachytene progression is inhibited in sperm-less animals, but only after oocytes have accumulated (Lopez et al., 2013; Miller et al., 2001). This suggests a chain of interdependent retrograde events in which an arrest in oocyte maturation first causes oocytes to accumulate, which then promotes GSC quiescence (and pachytene arrest), such that the assembly line stops producing more oocytes.

Alternatively, the absence of MSP signalling in the proximal somatic gonad could progressively block GSC proliferation independently of the arrest in oocyte maturation and accumulation. However, three pieces of evidence argue against this notion. First, neither of the gld-1 single mutants, gld-2 gld- 1 double mutants, or L1-upshifted fog-1; $g l p-1(g f)$ double mutants produce sperm (and MSP) or oocytes, but they all rapidly develop germline tumours. This indicates that they can maintain high GSC proliferation rates despite an absence of MSP signalling and further suggests that the presence of oocytes is required to generate an anti-proliferative signal when sperm is lacking. Second, in our oil diaphragm contraception experiment, we observed oocyte maturation in the injected arm, as evidenced by the endomitotic phenotype of proximal-most oocytes, indicating that MSP signalling was activated. Yet, the reactivation of MSP signalling and of oocyte maturation (at least in the proximalmost oocytes) was not accompanied by an increase in GSC proliferative activity and instead we observed that GSC quiescence was maintained. Third, oma-1; oma-2 double mutants have normal sperm and somatic MSP signalling, but accumulate oocytes and have a low GSC proliferative activity. Together, the evidence strongly suggests that MSP signalling is not sufficient to support elevated GSC proliferation activity, and that oocyte accumulation acts as the trigger for GSC quiescence.

Feedback regulation of stem cell activity has recently been observed in other circumstances (Hsu et al., 2014; Jiang et al., 2011; Mondal et al., 2011), and thus may represent a conserved feature of many stem cell systems. Whether localized IIS output neutralization is also conserved as the mechanism enabling such regulation remains to be determined. Our results also support the notion that only the accumulation of a specific terminally differentiated stem cell progeny, in this case oocytes, can efficiently exert a negative influence on stem cell proliferative activity. This suggests that a failure of stem cell progeny to fully or properly differentiate could perhaps underlie the aetiology of some hyper-proliferative diseases that are characterized by poor cellular differentiation, including psoriasis and certain types of cancer. In this view, the development of therapeutic regimes aimed at enhancing or mimicking terminal cell differentiation would be desirable.

\section{Germline-specific IIS counteraction}

Recent work demonstrated that the presence of males and mating have negative influences on a hermaphrodite's soma and life span (Maures et al., 2014; Shi and Murphy, 2014). Regarding the feedback regulation of GSCs, we note that the systemic downregulation of IIS dramatically extends the reproductive span (Luo et al., 2010); however, this is accompanied by a range of life-extending somatic side-effects that conversely impair sexual attractiveness, such as reduced motility, an abnormal appearance and aberrant pheromone secretion (Gems et al., 1998; Kuo et al., 2012). One appealing possibility is that germline-restricted IIS output control may have evolved to specifically delay reproductive ageing, or at least preserve germline integrity by slowing stem cell divisions, without otherwise compromising attractiveness. Such a local mechanism would increase the likelihood that a chance mating occurring later in life produces progeny, without risking missing this opportunity.

\section{MATERIALS AND METHODS}

\section{C. elegans genetics}

Animals were maintained at $15^{\circ} \mathrm{C}$ on standard $\mathrm{NGM}$ plates and fed Escherichia coli bacteria (OP50) unless otherwise indicated (Brenner, 1974). The Bristol isolate (N2) was used as wild type throughout. The following alleles, deficiencies and transgenes were used. LGI: goa-1(n1134), fog-1(q785), daf-16(mgDf47, mgDf50, mu86), gld-1(q485), gld-2(q497). LGIII: daf-2(e1370, e979), mpk-1(ga111), glp-1(ar202, e2141, q231, tn777), unc-79(e1068). LGIV: fem-3(q20), daf-18(nr2037), oma-1(zu405te33). LGV: him-5(e1490), fog-2(oz40, q71), spe-42(tn1231), acy-4(ok1806), oma-2(te51), ojIs1 [pie-1p::GFP::tbb-2; unc-119(+)]. LGX: muIs 109[daf-16p::GFP::DAF-16(+); odr-1p::RFP]. LG?: vizIs23 [pie-1p::GFP::DAF-2(+); unc-119(+)]. The following rearrangements were used. hT2[bli-4(e937) let-?(q782) qIs48](I;III), nT1 [unc-?(n754) let-? qIs50] (IV;V), nT1 [qIs51] (IV;V), nT1 [unc-?(n754) let-?] (IV;V). The following extra-chromosomal array was used: $\operatorname{tn} E x 37[a c y-4(+)$; sur-5p: GFP]

\section{GSC mitotic index evaluation}

For L3 and L4 stage animals, worms were synchronized by hatching in the absence of food and transferred to seeded plates at $25^{\circ} \mathrm{C}$. For adult animals, unless otherwise indicated, cultures were maintained at $15^{\circ} \mathrm{C}$, and mid-L4 stage (based on vulva development; Seydoux et al., 1993) animals were picked on a new seeded plate and transferred to $25^{\circ} \mathrm{C}$ for the indicated number of days with (mated) or without (unmated) him-5(e1490) or fog-2 (oz40) males. For continual mating experiments, small NGM plates were used with an approximate male:hermaphrodite ratio of 1:1 (typically 30 to 50 each) until the end of A6, when hermaphrodites usually stopped producing progeny, and males were removed. Hermaphrodites were transferred daily to new seeded plates, with or without young new males. For male GSC MI evaluation, him-5(e1490) L4 animals were staged based on spicule development (Baird and Ellazar, 1999) and picked to a new plate at $25^{\circ} \mathrm{C}$ until analysis. Gonads were dissected and stained as described below. 4'6-diamidino-2-phenylindole (DAPI) was used to highlight germ nuclei. Anti-HIM-3 antibodies were used to mark (and exclude) differentiating GSC progeny (HIM-3 positive), and anti-phospho[ser10]histone H3, to mark M-phase nuclei. As for delineating HIM-3 positive versus negative cells, we placed the boundary where all the cells in a crosssection (not necessarily a straight line) become HIM-3 positive. Our GSC numbers, where comparable, are similar to the published figures (Crittenden et al., 2006; Hansen and Schedl, 2013; Qin and Hubbard, 2015). Images of the distal germ line were acquired every $0.75 \mu \mathrm{m}$ using a $60 \times$ objective on a DeltaVision microscope. Undifferentiated germ nuclei counting in three dimensions was partially automated with an ImageJ plugin developed in Dr Jane Hubbard's laboratory, as described (Korta et al., 2012).

\section{Differentiation assays}

The $g l p-1(e 2141)$ allele is a temperature-sensitive loss of function of the Notch receptor, with $15^{\circ} \mathrm{C}$ being permissive, and $25^{\circ} \mathrm{C}$ being restrictive (Kodoyianni et al., 1992). Based on the previous observations that C. elegans development is roughly two times faster at $25^{\circ} \mathrm{C}$ (Byerly et al., 1976), we considered 2 days spent at $15^{\circ} \mathrm{C}$ to be equivalent to 1 day at $25^{\circ} \mathrm{C}$. Thus, A1 equivalent animals spent 2 days after the mid-L4 stage at $15^{\circ} \mathrm{C}$, 
and $\mathrm{A} 4$ equivalent animals spent 8 days at $15^{\circ} \mathrm{C}$, before being upshifted to $25^{\circ} \mathrm{C}$ for $15 \mathrm{~h}$. Experiments using the $g l p-1(q 231)$ and $g l p-1(t n 777)$ alleles used the same design, except that the animals were upshifted to $25^{\circ} \mathrm{C}$ for 8 (q231) and $24(\operatorname{tn} 777)$ hours before they were dissected and stained.

\section{Adult life span analyses}

Adult life span analyses were performed at $25^{\circ} \mathrm{C}$ in cohorts of $\sim 50$ animals as previously described (Narbonne and Roy, 2006b), excluding from analyses animals that died from internal hatching or dried on the edges of plates. For continual mating experiments, hermaphrodites were handled as described in the GSC mitotic index evaluation section.

\section{Staining}

Whole animals were fixed in Carnoy's solution and stained with DAPI as previously described (Narbonne and Roy, 2006b). For germline immunofluorescence, gonads were dissected out of the animal in a drop of PBS on a cover slip, which was placed against a poly-L-lysine coated slide, submitted to a freeze-crack and stained as previously described (Couteau et al., 2004). Primary mouse monoclonal anti-phospho[ser10]histone H3 (1:200; cat. no. 9706, Cell Signaling), rabbit polyclonal antiHIM-3 (1:500; a generous gift from Dr Monique Zetka) (Couteau et al., 2004), mouse anti-DAO-5 (1:25; Developmental Studies Hybridoma Bank clone DAO5) (Hadwiger et al., 2010), and secondary A488-conjugated goat anti-mouse, or A546-conjugated goat anti-rabbit antibodies (both at 1:500; Invitrogen) were used. DAPI was used as a counterstain.

\section{Oil diaphragm micro-injection}

Young adult hermaphrodites $(\angle \mathrm{A} 1)$ that were grown at $15^{\circ} \mathrm{C}$ were microinjected with a drop (of an approximate diameter of two to three oocytes) of mineral oil (Sigma) into either the anterior or posterior spermatheca and/or uterus, proximal to the first oocyte, using techniques previously described (Mello et al., 1991; Wolke et al., 2007). Animals were left less than 2 min on the injection pad, allowed to recover on a seeded plate for a few minutes, and transferred to a small patch of bacterial lawn containing fog-2(oz40) males at a $5: 1$ ratio at $25^{\circ} \mathrm{C}$ for $3 \mathrm{~h}$. Hermaphrodites were then singled on a new plate a $25^{\circ} \mathrm{C}$, verified for mating success by egg production (from the uninjected arm), and maintained until an obvious germline tumour appeared in at least one gonad arm, at which point animals were stained with DAPI and imaged. Animals that stopped moving normally or died prematurely were discarded from this analysis.

\section{Image acquisition and processing}

Images were acquired at a 0.5 (whole-worm) or 0.75 (germ line) $\mu \mathrm{m}$ interval using a $20 \times$ (whole-worm) or a $60 \times$ (germ line) objective on a DeltaVision microscope, deconvolved (whole-worm and distal germ lines), maximally projected, stitched (whole-worm), and thresholded using ImageJ. For display and ease of comparison purposes, whole-worms were computationally straightened using ImageJ

\section{Acknowledgements}

We are grateful to Jacques Boisvert, Joel Ryan, Abby Gerhold, Siegfried Hekimi, Richard Roy, Amy Maddox, David Greenstein, Philippe Roux, Marc Therrien, and the Labbé and Maddox laboratory members for their help, comments and/or suggestions. We also thank Swathi Arur, Monique Zetka, Carl Johnson, the CGC (funded by NIH Office of Research Infrastructure Programs P40 OD010440), Wormbase, and the C. elegans gene knockout consortium for providing strains and reagents.

\section{Competing interests}

The authors declare no competing or financial interests.

\section{Author contributions}

P.N. designed and conducted all the experiments, and wrote the manuscript, with edits from P.S.M. and J.-C.L.

\section{Funding}

P.N. was supported by a postdoctoral fellowship from the Human Frontier Science Program. This work was funded by a grant from the Canadian Institute of Health Research [MOP-115171 to P.S.M. and J.-C.L.]. P.S.M. is supported as a William Burwell Harrison Fellow of Biology. J.-C.L. holds the Canada Research Chair in Cell
Division and Differentiation. IRIC is supported in part by the Canada Foundation for Innovation and the Fonds de Recherche du Québec - Santé.

\section{Supplementary information}

Supplementary information available online at

http://dev.biologists.org/lookup/suppl/doi:10.1242/dev.130252/-/DC1

\section{References}

Angelo, G. and Van Gilst, M. R. (2009). Starvation protects germline stem cells and extends reproductive longevity in C. elegans. Science 326, 954-958.

Apfeld, J. and Kenyon, C. (1998). Cell nonautonomy of C. elegans daf-2 function in the regulation of diapause and life span. Cell 95, 199-210.

Arantes-Oliveira, N. (2002). Regulation of life-span by germ-line stem cells in Caenorhabditis elegans. Science 295, 502-505.

Austin, J. and Kimble, J. (1987). glp-1 is required in the germ line for regulation of the decision between mitosis and meiosis in C. elegans. Cell 51, 589-599.

Baird, S. E. and Ellazar, S. A. (1999). TGF $\beta$-like signaling and spicule development in Caenorhabditis elegans. Dev. Biol. 212, 93-100.

Barton, M. K., SchedI, T. B. and Kimble, J. (1987). Gain-of-function mutations of fem-3, a sex-determination gene in Caenorhabditis elegans. Genetics 115 , 107-119.

Berry, L. W., Westlund, B. and Schedl, T. (1997). Germ-line tumor formation caused by activation of glp-1, a Caenorhabditis elegans member of the Notch family of receptors. Development 124, 925-936.

Brenner, S. (1974). The genetics of Caenorhabditis elegans. Genetics 77, 71-94. Byerly, L., Cassada, R. C. and Russell, R. L. (1976). The life cycle of the nematode Caenorhabditis elegans: I. Wild-type growth and reproduction. Dev. Biol. 51 23-33.

Conboy, I. M., Conboy, M. J., Wagers, A. J., Girma, E. R., Weissman, I. L. and Rando, T. A. (2005). Rejuvenation of aged progenitor cells by exposure to a young systemic environment. Nature 433, 760-764.

Couteau, F., Nabeshima, K., Villeneuve, A. and Zetka, M. (2004). A component of C. elegans meiotic chromosome axes at the interface of homolog alignment, synapsis, nuclear reorganization, and recombination. Curr. Biol. 14, 585-592.

Crittenden, S. L., Leonhard, K. A., Byrd, D. T. and Kimble, J. (2006). Cellular analyses of the mitotic region in the Caenorhabditis elegans adult germ line. Mol. Biol. Cell 17, 3051-3061.

Dalfó, D., Michaelson, D. and Hubbard, E. J. A. (2012). Sensory regulation of the C. elegans germline through TGF- $\beta$-dependent signaling in the niche. Curr. Biol. 22, 712-719.

Detwiler, M. R., Reuben, M., Li, X., Rogers, E. and Lin, R. (2001). Two zinc finger proteins, OMA-1 and OMA-2, are redundantly required for oocyte maturation in C. elegans. Dev. Cell 1, 187-199.

Fox, P. M. and Schedl, T. (2015). Analysis of germline stem cell differentiation following loss of GLP-1 notch activity in Caenorhabditis elegans. Genetics 201, 167-184.

Fukuyama, M., Rougvie, A. E. and Rothman, J. H. (2006). C. elegans DAF-18/ PTEN mediates nutrient-dependent arrest of cell cycle and growth in the germline. Curr. Biol. 16, 773-779.

Gems, D., Sutton, A. J., Sundermeyer, M. L., Albert, P. S., King, K. V., Edgley, M. L., Larsen, P. L. and Riddle, D. L. (1998). Two pleiotropic classes of daf-2 mutation affect larval arrest, adult behavior, reproduction and longevity in Caenorhabditis elegans. Genetics 150, 129-155

Gerhold, A. R., Ryan, J., Vallée-Trudeau, J.-N., Dorn, J. F., Labbé, J.-C. and Maddox, P. S. (2015). Investigating the regulation of stem and progenitor cell mitotic progression by in situ imaging. Curr. Biol. 25, 1123-1134.

Govindan, J. A., Cheng, H., Harris, J. E. and Greenstein, D. (2006). Goo/i and Gos signaling function in parallel with the MSP/Eph receptor to control meiotic diapause in C. elegans. Curr. Biol. 16, 1257-1268.

Govindan, J. A., Nadarajan, S., Kim, S., Starich, T. A. and Greenstein, D. (2009). Somatic CAMP signaling regulates MSP-dependent oocyte growth and meiotic maturation in C. elegans. Development 136, 2211-2221.

Hadwiger, G., Dour, S., Arur, S., Fox, P. and Nonet, M. L. (2010). A monoclonal antibody toolkit for C. elegans. PLOS ONE 5, e10161.

Hansen, D. and Schedl, T. (2013). Stem cell proliferation versus meiotic fate decision in Caenorhabditis elegans. Adv. Exp. Med. Biol. 757, 71-99.

Hendzel, M. J., Wei, Y., Mancini, M. A., Van Hooser, A., Ranalli, T., Brinkley, B. R., Bazett-Jones, D. P. and Allis, C. D. (1997). Mitosis-specific phosphorylation of histone $\mathrm{H} 3$ initiates primarily within pericentromeric heterochromatin during $\mathrm{G} 2$ and spreads in an ordered fashion coincident with mitotic chromosome condensation. Chromosoma 106, 348-360.

Hsu, Y.-C., Li, L. and Fuchs, E. (2014). Transit-amplifying cells orchestrate stem cell activity and tissue regeneration. Cell 157, 935-949,

Iwasaki, K., McCarter, J., Francis, R. and Schedl, T. (1996). emo-1, a Caenorhabditis elegans Sec61p gamma homologue, is required for oocyte development and ovulation. J. Cell Biol. 134, 699-714.

Jiang, H., Grenley, M. O., Bravo, M.-J., Blumhagen, R. Z. and Edgar, B. A. (2011). EGFR/Ras/MAPK signaling mediates adult midgut epithelial homeostasis and regeneration in drosophila. Cell Stem Cell 8, 84-95. 
Johnson, T. E. (2006). Recent results: biomarkers of aging. Exp. Gerontol. 41 1243-1246.

Kadyk, L. C. and Kimble, J. (1998). Genetic regulation of entry into meiosis in Caenorhabditis elegans. Development 125, 1803-1813.

Kimble, J. and Ward, S. (1988). Germ-line Development and Fertilization. In The Nematode Caenorhabditis elegans (ed. W. B. Wood), pp. 191-213. Cold Spring Harbor, NY: Cold Spring Harbor Laboratory.

Kimura, K. D., Tissenbaum, H. A., Liu, Y. and Ruvkun, G. (1997). daf-2, an insulin receptor-like gene that regulates longevity and diapause in Caenorhabditis elegans. Science 277, 942-946.

Kodoyianni, V., Maine, E. M. and Kimble, J. (1992). Molecular basis of loss-offunction mutations in the glp-1 gene of Caenorhabditis elegans. Mol. Biol. Cell 3 1199-1213.

Korta, D. Z., Tuck, S. and Hubbard, E. J. A. (2012). S6K links cell fate, cell cycle and nutrient response in C. elegans germline stem/progenitor cells. Developmen 139, 859-870.

Kroft, T. L., Gleason, E. J. and L'Hernault, S. W. (2005). The spe-42 gene is required for sperm-egg interactions during $C$. elegans fertilization and encodes a sperm-specific transmembrane protein. Dev. Biol. 286, 169-181.

Kuo, T.-H., Fedina, T. Y., Hansen, I., Dreisewerd, K., Dierick, H. A., Yew, J. Y. and Pletcher, S. D. (2012). Insulin signaling mediates sexual attractiveness in Drosophila. PLoS Genet. 8, e1002684.

Lackner, M. R. and Kim, S. K. (1998). Genetic analysis of the Caenorhabditis elegans MAP kinase gene mpk-1. Genetics 150, 103-117.

LaFever, L. and Drummond-Barbosa, D. (2005). Direct control of germline stem cell division and cyst growth by neural insulin in Drosophila. Science 309, 1071-1073

Leacock, S. W. and Reinke, V. (2006). Expression profiling of MAP kinasemediated meiotic progression in Caenorhabditis elegans. PLoS Genet. 2, e174.

Lee, R. Y. N., Hench, J. and Ruvkun, G. (2001). Regulation of C. elegans DAF-16 and its human ortholog FKHRL1 by the daf- 2 insulin-like signaling pathway. Curr Biol. 11, 1950-1957.

Lee, M.-H., Ohmachi, M., Arur, S., Nayak, S., Francis, R., Church, D., Lambie, E. and Schedl, T. (2007). Multiple functions and dynamic activation of MPK-1 extracellular signal-regulated kinase signaling in Caenorhabditis elegans germline development. Genetics 177, 2039-2062.

Lin, K., Hsin, H., Libina, N. and Kenyon, C. (2001). Regulation of the Caenorhabditis elegans longevity protein DAF-16 by insulin/IGF-1 and germline signaling. Nat. Genet. 28, 139-145.

Lopez, A. L., III, Chen, J., Joo, H.-J., Drake, M., Shidate, M., Kseib, C. and Arur, S. (2013). DAF-2 and ERK couple nutrient availability to meiotic progression during Caenorhabditis elegans oogenesis. Dev. Cell 27, 227-240.

López-Otín, C., Blasco, M. A., Partridge, L., Serrano, M. and Kroemer, G. (2013). The hallmarks of aging. Cell 153, 1194-1217.

Luo, S., Kleemann, G. A., Ashraf, J. M., Shaw, W. M. and Murphy, C. T. (2010) TGF- $\beta$ and insulin signaling regulate reproductive aging via oocyte and germline quality maintenance. Cell 143, 299-312.

Maures, T. J., Booth, L. N., Benayoun, B. A., Izrayelit, Y., Schroeder, F. C. and Brunet, A. (2014). Males shorten the life span of C. elegans hermaphrodites via secreted compounds. Science 343, 541-544.

Mello, C. C., Kramer, J. M., Stinchcomb, D. and Ambros, V. (1991). Efficient gene transfer in C.elegans: extrachromosomal maintenance and integration of transforming sequences. EMBO J. 10, 3959-3970.

Michaelson, D., Korta, D. Z., Capua, Y. and Hubbard, E. J. A. (2010). Insulin signaling promotes germline proliferation in C. elegans. Development 137 $671-680$
Miller, M. A., Nguyen, V. Q., Lee, M.-H., Kosinski, M., Schedl, T., Caprioli, R. M. and Greenstein, D. (2001). A sperm cytoskeletal protein that signals oocyte meiotic maturation and ovulation. Science 291, 2144-2147.

Mondal, B. C., Mukherjee, T., Mandal, L., Evans, C. J., Sinenko, S. A., MartinezAgosto, J. A. and Banerjee, U. (2011). Interaction between differentiating celland niche-derived signals in hematopoietic progenitor maintenance. Cell 147, 1589-1600.

Morgan, D. E., Crittenden, S. L. and Kimble, J. (2010). The C. elegans adult male germline: Stem cells and sexual dimorphism. Dev. Biol. 346, 204-214

Morris, G. M., van den Aardweg, G. J. M. J., Hamlet, R., Whitehouse, E., Hopewell, J. W., Franke, H. and Loeffler, M. (1990). Age-related changes in the cell kinetics of rat foot epidermis. Cell Proliferation 23, 113-123.

Morrison, S. J. and Kimble, J. (2006). Asymmetric and symmetric stem-cell divisions in development and cancer. Nature 441, 1068-1074.

Murphy, C. T. and Hu, P. J. (2013). Insulin/insulin-like growth factor signaling in C. elegans. WormBook 1-43.

Murphy, C. T., Lee, S.-J. and Kenyon, C. (2007). Tissue entrainment by feedback regulation of insulin gene expression in the endoderm of Caenorhabditis elegans. Proc. Natl. Acad. Sci. USA 104, 19046-19050.

Narbonne, P. and Roy, R. (2006a). Regulation of germline stem cell proliferation downstream of nutrient sensing. Cell Div. 1, 29

Narbonne, P. and Roy, R. (2006b). Inhibition of germline proliferation during C. elegans dauer development requires PTEN, LKB1 and AMPK signalling. Development 133, 611-619.

Narbonne, P., Hyenne, V., Li, S., Labbé, J.-C. and Roy, R. (2010). Differential requirements for STRAD in LKB1-dependent functions in C. elegans. Development 137, 661-670.

Ogg, S. and Ruvkun, G. (1998). The C. elegans PTEN homolog, DAF-18, acts in the insulin receptor-like metabolic signaling pathway. Mol. Cell 2, 887-893.

Pepper, A. S.-R., Killian, D. J. and Hubbard, E. J. A. (2003). Genetic analysis of Caenorhabditis elegans glp-1 mutants suggests receptor interaction or competition. Genetics 163, 115-132.

Pickett, C. L., Dietrich, N., Chen, J., Xiong, C. and Kornfeld, K. (2013). Mated progeny production is a biomarker of aging in Caenorhabditis elegans. G3 3 2219-2232.

Pinkston, J. M., Garigan, D., Hansen, M. and Kenyon, C. (2006). Mutations that increase the life span of C. elegans inhibit tumor growth. Science 313, 971-975.

Qin, Z. and Hubbard, E. J. A. (2015). Non-autonomous DAF-16/FOXO activity antagonizes age-related loss of $\mathrm{C}$. elegans germline stem/progenitor cells. Nat Commun. 6, 7107

Seydoux, G., Salvage, C. and Greenwald, I. (1993). Isolation and characterization of mutations causing abnormal eversion of the vulva in Caenorhabditis elegans. Dev. Biol. 157, 423-436.

Shi, C. and Murphy, C. T. (2014). Mating induces shrinking and death in Caenorhabditis mothers. Science 343, 536-540.

Shim, J., Gururaja-Rao, S. and Banerjee, U. (2013). Nutritional regulation of stem and progenitor cells in Drosophila. Development 140, 4647-4656.

Sousa-Victor, P., Gutarra, S., García-Prat, L., Rodriguez-Ubreva, J., Ortet, L., Ruiz-Bonilla, V., Jardí, M., Ballestar, E., González, S., Serrano, A. L. et al. (2014). Geriatric muscle stem cells switch reversible quiescence into senescence. Nature 506, 316-321.

Tomasetti, C. and Vogelstein, B. (2015). Variation in cancer risk among tissues can be explained by the number of stem cell divisions. Science 347, 78-81.

Wolke, U., Jezuit, E. A. and Priess, J. R. (2007). Actin-dependent cytoplasmic streaming in C. elegans oogenesis. Development 134, 2227-2236.

Zetka, M. (2009). Homologue pairing, recombination and segregation in Caenorhabditis elegans. Genome Dyn. 5, 43-55. 